\title{
Drosophila melanogaster as an alternative model organism in nutrigenomics
}

Nieves Baenas ${ }^{1}$ and Anika E. Wagner ${ }^{2^{*}}$ (i)

\begin{abstract}
Nutrigenomics explains the interaction between the genome, the proteome, the epigenome, the metabolome, and the microbiome with the nutritional environment of an organism. It is therefore situated at the interface between an organism's health, its diet, and the genome.

The diet and/or specific dietary compounds are able to affect not only the gene expression patterns, but also the epigenetic mechanisms as well as the production of metabolites and the bacterial composition of the microbiota. Drosophila melanogaster provides a well-suited model organism to unravel these interactions in the context of nutrigenomics as it combines several advantages including an affordable maintenance, a short generation time, a high fecundity, a relatively short life expectancy, a well-characterized genome, and the availability of several mutant fly lines. Furthermore, it hosts a mammalian-like intestinal system with a clear microbiota and a fat body resembling the adipose tissue with liver-equivalent oenocytes, supporting the fly as an excellent model organism not only in nutrigenomics but also in nutritional research. Experimental approaches that are essentially needed in nutrigenomic research, including several sequencing technologies, have already been established in the fruit fly. However, studies investigating the interaction of a specific diet and/or dietary compounds in the fly are currently very limited. The present review provides an overview of the fly's morphology including the intestinal microbiome and antimicrobial peptides as modulators of the immune system. Additionally, it summarizes nutrigenomic approaches in the fruit fly helping to elucidate host-genome interactions with the nutritional environment in the model organism Drosophila melanogaster.
\end{abstract}

Keywords: Drosophila melanogaster, Nutrigenomics, Diet, Model organism

\section{Background}

Nutrigenomics defines the nutrient-gene interactions in a host and at present includes not only nutrient-gene interactions but also nutrient-epigenetic, nutrient-proteomic, and nutrient-metabolomic interactions as well as host-diet-microbiome interactions [1]. In this sense, nutrigenomic research is located on the intersection between diet, health, and genomics [2, 3].

Drosophila melanogaster is a model organism essentially applied in genetic research that brings promising advantages into studying preclinical nutrigenomics. Its evolutionary biology significantly contributes to the understanding of gene expression and development in humans, as its genome conserves approximately $60 \%$ of

\footnotetext{
*Correspondence: anika.wagner@uni-giessen.de

${ }^{2}$ Institute of Nutritional Sciences, Justus-Liebig-University, Wilhelmstrasse 20,

35392 Giessen, Germany

Full list of author information is available at the end of the article
}

genes that are related with DNA mutations, amplifications, or deletions in a diverse set of human diseases [4, 5]. Its genome encodes ca. 18,000 genes located on four homologous pairs of chromosomes, while only three of them hold the main part of the genome. Flies and mammalian species normally share about $40 \%$ of the nucleotide and protein sequences in their homologs; in some conserved functional domains, it can be more than $90 \%$ $[6,7]$. Chromosomal deletions and mutations have been generated for the production of Drosophila melanogaster mutants, targeting more than $80 \%$ of its genome [8].

Beside its well-characterized genome and the good availability of mutant and transgenic flies, other advantages including a rapid life cycle (12 days for the succession of egg, maggot, pupa, and imago), a short life span (around 70-80 days), a small size (possibility of breeding hundreds of individuals in small bottles), and a relatively easy generation of mutant animals in comparison to

(C) The Author(s). 2019 Open Access This article is distributed under the terms of the Creative Commons Attribution 4.0 International License (http://creativecommons.org/licenses/by/4.0/), which permits unrestricted use, distribution, and 
other organisms make Drosophila melanogaster an excellent model organism in nutrigenomic research.

Particularly, due to the presence of a fat body with adipocytes and conserved metabolic pathways involved in fat metabolism and insulin signaling, Drosophila melanogaster has been extensively used to investigate obesity-associated diseases, including cardiovascular dysfunction or cancer [9-11]. Changes in triglyceride levels and lipid storage induced by the intake of high-fat and high-sugar diets have been related with genetic variations in both genes of the insulin/insulin-like growth factor signaling (IIS) and the target of rapamycin (TOR) signaling pathway $[12,13]$.

The fruit fly also resembles a good model to study different tissues or organs due to its mammalian-like anatomy and equivalent functions. The present review provides information on the fruit fly's morphology and anatomy with a special focus on the gastrointestinal system and the gut microbiota, key facts in nutrigenomics studies. Additionally, it gives insights into the different methods applied in nutrigenomics and their utilization in Drosophila melanogaster.

\section{Drosophila melanogaster-morphology}

Drosophila melanogaster presents different morphology traits as a product of natural selection. These differences are generally associated with gene mutations referring to single phenotypes [14]. Mutation markers indicating differences in bristles, wings, appendages, eye shapes, and colors and body sizes have been collected by FlyBase (www.flybase.org), providing accurate information about its location in the chromosomes. Environmental factors, such as nutrition, temperature, or crowding, have been reported to be responsible for morphological traits, in particular, body size variations, and have been connected to quantitative trait loci (QTL) mapping on the third chromosome while no QTLs or QTLs with minor effects on these factors have been detected in the other major chromosomes [14]. The time for fly development, also known as the fruit fly life cycle, varies within different environmental conditions. Generally, the development of new flies takes around 10 days at $25^{\circ} \mathrm{C}$ with four developmental stages: the embryo, larvae (three different stages), pupal stage, and imago stage. The adult flies reach sexual maturity 2-4 days after eclosion.

According to a typical insect morphology, the adult fruit fly body is divided into three parts: head, thorax, and abdomen. In the head, there are several sensory organs, noting the compound eyes, containing primary pigments being characteristic for different mutants, and the proboscis, representing the gustatory organ for food detection, taste, and intake, which could be extended and retracted and pumps the food into the gut. The thorax is divided into three sections: prothorax (anterior) with one pair of legs, mesothorax (middle) with one pair of legs and one pair of wings, and metathorax (posterior) with one pair of legs and one pair of halters (modified wings). Females and males can be easily differentiated by morphological attributes, especially, females are generally bigger and possess an abdomen that has a pointed tip whereas males show a rounded abdomen with black pigmentation in the posterior segment with an epandrium (male external genitalia) [15].

The anatomy of the fly includes organ systems with equivalent functions to mammalian organisms, including the brain, peripheral nervous system, heart, trachea system (similar to the lung), esophagus, Malpighian tubules (similar to the kidneys), fat body with oenocytes (combining the functions of adipose tissue and the liver), gut, and gonads [16]. The fly brain possesses more than 100,000 neurons and exhibits important functions in a similar way as in the mammalian central nervous system, including circadian rhythms, sleep, learning, memory, courtship, feeding, aggression, grooming, and flight navigation. Therefore, this model organism offers the possibility to investigate feeding-associated behaviors by analyzing metabolic changes in conjunction with neuroendocrine and neuromodulatory states and underlying molecular mechanisms [17]. It has been documented that flies react to various dietary compounds or drugs within their central nervous system in a similar way as observed in mammalian systems [6].

Regarding the significant importance of the digestive tract in the context of nutrition research, the present review provides detailed information on the digestive tract including its microbiota. Drosophila melanogaster's alimentary canal consists of a simple epithelium which encompasses visceral muscles, trachea, and nerves [18]. Depending on their position along the gut length, these different cell types differ in their arrangement and functions which may vary on their different developmental origins [18]. The intestinal epithelium of the fruit fly consists of a monolayer of four different types of cells: intestinal stem cells (ISC), absorptive enterocytes (EC), secretory enteroendocrine (EE) cells, and enteroblasts (EB). The last may differentiate either into an EC or an EE depending on the different signals present in specific parts of the fly's digestive tract [19]. Under normal physiological conditions, the ISC proliferate and differentiate in a rate that maintains a correct intestinal barrier function [20]. During aging, proliferation and differentiation of these ISC may be impaired resulting in epithelial dysplasia [21]. A loss of ISC, a disturbed epithelial turnover, and an impaired epithelial ultrastructure have been suggested to cause a decrease in Drosophila melanogaster life span following the uptake of the probiotic strain Lactobacillus plantarum [20]. These results are in contrast to other studies showing beneficial effects 
of $L$. plantarum especially in the context of developmental rates and ISC proliferation in young Drosophila melanogaster [22-26]. This suggests that the effects of health-promoting gut microbes may also depend on various factors including age and genotype as well as the applied probiotic strains and the diet [26].

The fly's alimentary canal is roughly divided into foregut, midgut, and hindgut [27]; while the foregut is of ectodermal origin, the midgut and the hindgut are-as all other organs of the fly-of endodermal origin [28]. Specifically, the foregut consists of the mouth, the pharynx, the esophagus, and the crop [29], an organ for the storage and mixing of food, as well as for the detoxification. The foregut is connected with the midgut by the cardia, a sphincter that controls the food passage [27]. The midgut is the central part of the digestion as digestive enzymes are excreted and nutrients are absorbed [30]. Historically, the midgut has been further divided into the anterior, middle, and posterior part, while it has been recently classified into six different anatomical regions (R0-R5) exhibiting specific metabolic and digestive functions [30]. Within the midgut, a region with a $\mathrm{pH}$ of $<4.0$ exists, indicating that the so-called copper cells secrete acid-like the parietal cells in the mammalian stomach - which in consequence helps to digest proteins [18] and supports the permanent colonization of the alimentary tract with commensal bacteria [21]. Similar to the mammalian mucus layer, the midgut of the fly is lined by a peritrophic matrix (PM) that is produced by the crop and is composed of glycoproteins and chitin, potentially protecting the midgut epithelium from harmful particles and microbes [29].

\section{Drosophila melanogaster-microbiota}

In humans, the gastrointestinal tract is populated by a multiplicity of microorganisms including more than 500 different bacterial species. In the present context, the so-called microbiota refers to the commensal bacteria present in the colon [31]. In healthy human subjects, the microbiota shows a distinguished composition that consists of five phyla: mainly Bacteroidetes (Bacteroides ssp.) and Firmicutes (Lactobacillus spp.), and also Actinobacteria (Bifidobacterium ssp.), Proteobacteria (Escherichia, Helicobacter), and Verrucomicrobia (Akkermansia spp.) $[32,33]$. This microbiota composition is vulnerable during childhood and advanced age and rather stable during adulthood [33]. Various studies have reported a high microbiota diversity between subjects suggesting an association with different diets and obesity and consequently in energy homeostasis [32].

Taking advantage of the sophisticated genetic tools available in the fruit fly Drosophila melanogaster, its complex gastrointestinal system and the presence of a clear microbiota, it would be a predestined model to unravel host-microbiota interactions related to nutrition. The gut of Drosophila melanogaster hosts a limited number of commensal gut bacteria ranging from 3 to 30 species, including Lactobacillus plantarum as the most prevalent, Acetobacter pomorum, A. tropicalis, L. frucitvorans, and $L$. brevis $[34,35]$. Interestingly, populations of Lactobacillus species are common to both fly midguts and animal small intestines [36] and have been associated with several biological functions in Drosophila melanogaster, including larval growth, food uptake, and protection from malnutrition or oxidative stress, similar to health-promoting properties of Lactobacillus in mammals [37]. Laboratory fly stocks are associated with a relatively low number of taxa (about 1-13 OTUs define around $97-99 \%$ of identity, depending on the study), while most bacteria refer to two genera: Acetobacter and Lactobacillus [38]. Large changes in both microbial load and composition of bacterial species in the Drosophila intestinal microbiota are - similar to humans-more closely associated with the animal's gut morphology, epithelial architecture, and health status than with its chronological age [23]. It is not astonishing that some studies have reported different bacterial compositions in the gut of wild and laboratory strains of Drosophila melanogaster, supporting the assumption that the microbiota mainly corresponds to bacteria growing on the ingested foods and rather needs a permanent and repeated ingestion through the diet to permanently colonize the fly's intestine [39, 40]. Similarly, Pais et al. (2018) reported that laboratory stocks $\left(\mathrm{w}^{1118}\right)$ host mainly two bacterial species in their gut corresponding to Acetobacter OTU2753 and Lactobacillus OTU1865, which, however, cannot persist in the gut without a reinfection via their foods. Interestingly, in wild-caught fruit flies, 35 different OTUs, corresponding to Enterobacteriaceae, Acetobacteriaceae (mainly Acetobacter and Gluconobacter species), Leuconostocaceae, and Bacillaceae, were identified as the most prevalent families, partly containing bacterial strains that are able to stably colonize the fly gut, such as $L$. pseudomesenteroides, $A$. cibinongensis, and A. thailandicus [41]. Therefore, a further characterization of the host's interaction with persistent gut-colonizing bacteria would contribute to a better understanding in the context of Drosophila-microbe interactions. Nevertheless, several studies have demonstrated a significant impact of Drosophila gut commensal microbes on host-signaling pathways, metabolic capacities, development, locomotion, immune response, intestinal functionality, and aging, demonstrating that an excessive bacterial growth or dysbiosis promotes the organism's death [42].

Sterile or axenic fly strains (reared under germfree conditions) may be generated either by applying low doses of streptomycin to the diet or by performing egg 
dechorionation [43]. To obtain flies with a defined microbial community (gnotobiotic flies), flies will either be exposed to correspondingly inoculated sterile diets or embryos will encounter microbial species of interest [44]. In an experiment using axenic and gnotobiotic flies, Dobson et al. [45] compared the co-expression of specific and functionally related genes associated with growth, metabolism, and neurophysiological regulators (such as the components of the IIS and TOR pathways), showing an upregulation of these genes in the presence of the microbiota, and consequently its influence on the host transcriptome [45]. A recent publication demonstrated that the elimination of the microbiota altered the expression of immune response-associated genes, as well as genes connected with oxidative stress and general detoxification, in the head of young adult Drosophila melanogaster [46].

\section{Nutrigenomic approaches in Drosophila melanogaster}

As mentioned earlier, nutrigenomics refers not only to gene-nutrient interactions but also to nutrient-epigenetic, nutrient-proteomic, nutrient-metabolomic, and nutrientmicrobiome interactions (Fig. 1).

Diverse transcriptomic tools may be used in nutrigenomics research in Drosophila melanogaster including microarrays, to deliver information on changes in the mRNA expression following the dietary intake of a specific nutrient [7], and RNA sequencing [10] and next-generation sequencing (NGS) technologies [47], to analyze regions of interest in the genome, providing promising results and solutions to nutrigenomics studies by identifying new mutations in inbred fly strains. In addition, studies of QTL [48], representing a genome region that causes a significant variation in a quantitative trait, may be used in identifying signaling pathways involved in the metabolism of specific nutrients. An important goal achieved in Drosophila genetics research is the generation of an RNAi knockdown fly line collection by the Vienna Drosophila Research Centre, targeting around $90 \%$ of the whole fly genome and being accessible for the research community [6]. Until then, large-scale RNAi screens of gene function have been mainly performed in Caenorhabditis elegans, although it exhibits systemic RNAi for which reason the gene interference cannot be referred to a specific cell type [49]. As RNAi of Drosophila melanogaster is cell autonomous, it can be activated by inserting a transgenic long doublestranded "hairpin" RNA [49]. By combining this tool with the GAL4/UAS system in Drosophila, it offers the possibility to inactivate the expression of a specific gene in various different cell types helping to generate conditional transgenic fly models [50]. This makes it easier to study the overexpression or the misexpression of fly homologous genes and proteins, helping to establish fly models to study human diseases.

\section{Genomics}

The genome refers to the genetic material of an organism consisting of DNA. Genes (coding regions of the DNA) and non-coding regions of the DNA, mitochondrial DNA as well as chloroplast DNA, are parts of the genome [51]. The Drosophila genome has a size of 180

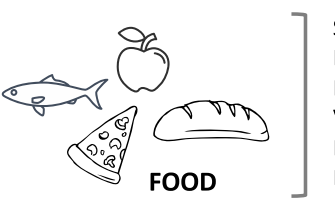

$\begin{array}{ll}\text { Sugar } & \text { (Carbohydrate } \\ \text { Lipids } & \text { (Fatty Acids) } \\ \text { Proteins } & \text { (Amino Acids) } \\ \text { Vitamins } & \\ \text { Minerals } & \\ \text { Bioactive compounds }\end{array}$

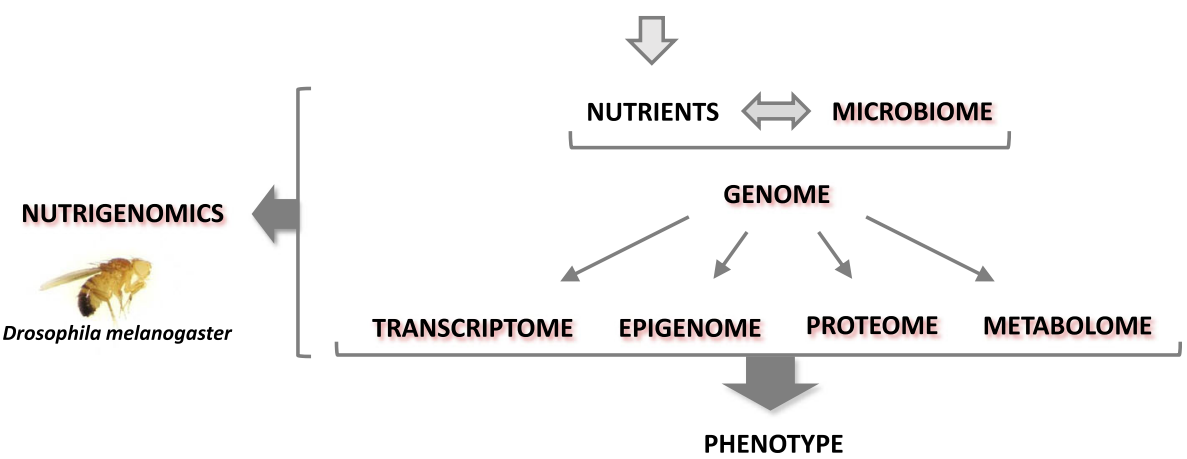

Fig. 1 Overview of the nutrigenomics approach in the model organism Drosophila melanogaster. An organism ingests complex foods which are degraded into nutrients that interact with the microbiome. This in consequence affects the genome, involving the transcriptome, the epigenome, the proteome, and the metabolome, resulting in the organism's phenotype. The fruit fly can be used as a model organism in nutrigenomics, as changes in the microbiome, transcriptome, epigenome, proteome, and metabolome due to an interaction with the nutritional environment are detectable and evaluable by several methods (pictograms used are from vecteezy.com) 
$\mathrm{Mb}$ and is packed into four pairs of chromosomes, and the genome sequence has been known for nearly 20 years [52]. Each of the large chromosomes contains a DNA molecule with $5 \mathrm{~cm}$ in length that has to fit into a nucleus with a diameter of ca. $5 \mu \mathrm{m}$. This indicates that the chromosomes need to be condensed several thousand times to fit perfectly into the small nucleus which is mediated by chromatin folding. During the last decades, it has become obvious that this DNA organization essentially contributes to the regulation of the gene expression which is referred to as epigenetic regulation [52].

\section{Transcriptomics}

The transcriptome refers to all messenger RNAs present in one cell or a population of cells at a defined time [53]. The analysis of the transcriptome has been mainly dominated by microarray analysis provided by different companies, including Affymetrix, Agilent Technologies, and Illumina. Recently, these analyses have been based on the RNAseq technology, defined as transcriptome profiling using NGS. It is stated that this methodology offers-compared to microarrays-the advantage of the detection of lower abundant and wider ranges of transcripts [54]. By comparing the intake of two different obesogenic diets, RNAseq analysis from Drosophila heads revealed significant differences in the transcriptome. While genes associated with immunity, metabolism, and hemocyanin have been mainly affected in flies fed with a high-fat diet, genes connected with cell cycle checkpoint kinases (CHK), cell cycle activity, and DNA binding and transcription have been upregulated in flies receiving a high-sugar diet [10]. In a recent study by Azuma and colleagues [55], plant bioactives have been applied to detect antiobesogenic effects in a fly model of obesity. RNAseq analysis has been performed to detect differentially regulated genes in male and female flies fed with a coconut-oil-supplemented high-fat diet, either in the presence or in the absence of quercetin glycosides (QG) or epigallocatechin gallate (EGCG). This is-as far as we know-one of the first publications presenting lists of differentially regulated genes in obese flies using RNAseq data analysis. These results have been supported by functional analysis showing lower triglyceride levels in flies under QG or EGCG supplementation. This study, as well as our own experiment demonstrating a clear visual separation of the fly's transcriptome following a dietary supplementation of the secondary bile acid lithocholic acid (LCA), pushes the fruit fly as an excellent model organism in nutrition research and, specifically, in the context of transcriptomic analysis. Gene set enrichment analysis has shown a downregulation of TOR, metabolism, Wnt, p53, and immune processes, whereas genes associated with the cell cycle have been increased following dietary LCA treatment [56]. An earlier study by Ye and colleagues [7] performed transcriptomic analysis by using the microarray technology. Preliminary results have been generated in flies being exposed to different energy sources in their diets, including sucrose as a control, palmitic acid, soy, and beef. Changes in the gene expression levels of ca. 2-3\% within the ca. 18,000 genes have been observed following the intake of the different diets [7]. Additionally, in Drosophila larvae, a starvation of amino acids changed the transcriptome, especially metabolism-associated genes, mainly involved in the TOR pathway [57].

\section{Epigenetics}

The term epigenetics defines heritable phenotype alterations which are not mediated by a change in the DNA sequence. Epigenetic changes are mediated by histone modifications, DNA methylation, and microRNA expressions [58]. The epigenome changes within the cells and is more dynamic compared to the genome [59]. It has been documented that our diet is able to induce epigenetic alterations that, in consequence, affect biomarkers of metabolic modulations in different model organisms as well as in human subjects. A very famous example of epigenetic effects due to dietary changes are humans that survived the so-called Dutch hunger winter in 1944 [60]. Several years later, researchers were able to detect changes in different metabolic markers in their offspring, such as the glucose tolerance [61], which resulted from a change in the methylation pattern of specific genes due to a limited availability of calories during the gestational period $[62,63]$. To detect epigenetic changes in a biological sample, MethyLight technology, pyrosequencing, chromatin immunoprecipitation-on-chip (ChIP-on-chip), and quantitative methylation-specific polymerase chain reaction (QMSP) followed by pyrosequencing can be applied [59]. All methods use the sodium bisulfite treatment as the compound reacts with unmethylated cytosine and converts it into uracil, which helps to deliver information on DNA methylation via PCR technology [59]. The detection of changes in microRNA expression is mainly performed by gene-chip microarray technology (Affymetrix), while histone modifications are detected by applying specific monoclonal antibodies against histone modifications or by a ChIP-seq assay followed by NGS [59].

Studying diet-related effects on epigenetic mechanisms in fruit flies has just recently started [64, 65]. The administration of diets with a varying macronutrient composition shows persistent changes of genes associated with epigenetic mechanisms over generations [64]. A study by Lian and co-workers [65] looked into the DNA methylation pattern of flies reared under dietary restriction. Unexpectedly, the methylome of these flies exhibited only minor changes which may be due to the 
relatively young age (7 days) at the sampling day as changes in the life span due to dietary restriction usually occur at a later time point [66]. Further research looking into DNA methylation pattern in flies under dietary restriction at an older age would therefore provide more valuable data regarding epigenetic modulations. Another possibility to check epigenetic changes is to study chromatin remodeling. In this regard, Sebald and colleagues demonstrated a central role of the chromatin remodeling factor CHD1 on a healthy microbiome composition in the fruit fly [67], which indirectly indicates an effect of the diet, as it is the most prominent factor affecting the intestinal commensal bacteria $[68,69]$. This study exemplified the fruit fly as an upcoming model organism in epigenetic research, helping to elucidate diet-dependent effects on the epigenome. In the context of epigenetic research, the fruit fly offers the advantage to investigate epigenetic effects throughout different generations during a relatively short period of time.

Other molecules that epigenetically modify gene expression are microRNAs (miRNA), small non-coding RNAs with a length of 17-25 nucleotides, normally inhibiting gene expression. Their main type of action is via (a) an inhibited translation and/or (b) by inducing the degradation of the mRNAs, known to be centrally involved in the epigenetic regulation of gene expression [70]. MicroRNAs play a central role in cellular processes such as proliferation, differentiation, and apoptosis, which are known pathways affected in the development of chronic diseases including cancer [71]. Studies have shown that especially plant bioactives are able to affect miRNA expression which may partly explain their health-promoting properties documented in the development of various chronic inflammatory diseases [72-74]. Initial experiments identified lin-4 as the first miRNA being essential for the normal development of Ceanorhabditis elegans [75]. Drosophila melanogaster has also been successfully used to generate essential information on effects of miRNA, by establishing the Flp-FRT and GAL4-UAS systems, allowing to knock-in or knock-out specific miRNAs with particular functions in the fly [71]. The state-of-the-art technology CRISPR/Cas9 has been recently established in the context of miRNA research as miR-219 and miR-315 have been successfully knocked down in Drosophila melanogaster [76]. This fact points towards an important input in elucidating miRNA-based processes [71]. In addition, it offers the possibility to use the fruit fly as a model organism to elucidate health-promoting or health-declining effects of different macronutrients and/or specific food components potentially related to miRNA modulation.

\section{Proteomics}

The proteome is defined as the protein complement that is present in a cell, an organ, or an organism at a given time $[54,77]$. As proteins present the functional part of genes and the mRNA information, the proteome accounts for the organism's phenotype [77]. Data regarding the proteome of Drosophila melanogaster in connection to different diets and/or dietary compounds are currently very limited. $\mathrm{Li}$ and co-workers demonstrated a change in the midgut proteome of the fruit fly receiving the Bowman-Birk protease inhibitor via their diet [78]. In comparison to control diet-fed animals, the proteomic analysis in fly larvae exposed to this inhibitor showed an impaired expression of proteins associated with protein degradation and transport, as well as fatty acid catabolism [78]. Another study investigated the effect of dietary ethanol on the proteome of fruit flies. Culwell and colleagues have detected relatively stable proteomes following the treatment with $10 \%$ ethanol compared to control-fed flies [79]. Admittedly, the authors have only focused on short-term effects of the applied compound with the intention to confirm the so-called Hamburger effect, which has been suggested for human proteomes following the consumption of one single hamburger [80].

In addition, antimicrobial peptides, including metchnikowin, diptericin, attacins, cecropinA1, and drosocin, have been widely used as biomarkers for the Drosophila melanogaster immune system, playing a crucial role in the defense mechanisms, the stem cell proliferation, and the regulation of the gut microbiota in mammals [47]. The identification and quantification of different antimicrobial peptides by mass spectrometry technologies and gel electrophoresis, as well as their expression levels using qRT-PCR and NGS, may be evaluated to get information on the health status and especially on the immune status of Drosophila melanogaster receiving different diets or supplements such as bioactive compounds. Altered anti-microbial peptide levels have been related to an impaired proliferation of ISC and intestinal bacterial loads. In particular, an increased expression of the antimicrobial peptides drosocin and cecropin A1 in the intestine has been connected with a prolonged life span of flies [81]. This increased expression of drosocin and cecropin A1 is associated with a lower activation of the classical immune pathways in the midgut of these flies, such as the immune deficiency (IMD) and Janus kinase-signal transducers and activators of transcription (JAK-STAT) pathway, as well as with lower activities of c-Jun N-terminal kinase (JNK) and epidermal growth factor (EGF) which points towards a better regeneration and maintenance of ISC and an alleviated stress response [81]. In a recent publication, Hanson and colleagues [82] used flies lacking all 14 antimicrobial peptides, that have been systematically tested for their effects on Gram-positive and Gram-negative bacteria and fungi. The Drosophila antimicrobial peptides mainly 
affect Gram-negative bacteria and represent rather effectors than regulators of the innate immune system in the fruit fly [82]. Effectors are built in an immune reaction with an antigen while regulators mainly repress ongoing immune reactions.

Although only limited information of diets and/or specific nutrients/nutritional factors on the fly proteome are currently available, the fruit fly could be a suitable model organism to unravel effects of specific diets/nutrients/ bioactive ingredients on the protein expression. Methods to detect alterations of the proteome include (a) methods to separate the proteins and (b) methods to identify and characterize the proteins. Extractions, precipitations, chromatography, electrophoresis, and centrifugation can be applied to separate the proteins, while mass spectrometry, nuclear magnetic resonance (NMR) spectroscopy, and immune labeling can be used for protein identification and characterization.

\section{Microbiomics}

The gut microbiota in the fruit fly can be isolated after the dissection of the gut or from the whole fly [23]. By using the whole fly, usually, the surface is disinfected by ethanol in order to remove external bacteria. In addition, a non-invasive approach can be applied by collecting and analyzing fecal spots that have been deposited by the flies during a defined period [83]. This offers the advantage of analyzing microbiota dynamics in the same cohort at several time points, like throughout a life span experiment or nutritional interventions. As far as we know, there are only a few studies available in Drosophila melanogaster that have analyzed the microbiota composition after applying a specific diet or a specific dietary compound. Recently, Erkosar et al. [84] have demonstrated a drastic effect on the abundance and the $\alpha$-diversity of the intestinal microbiota in fruit flies following the intake of specific nutrients. The authors observed a 100-fold induction in the total abundance of bacterial members of the Drosophila microbiota by increasing dietary yeast from 4 to $27 \%$ in the fly food [84]. In another study, the bioactive compound ursolic acid has been added to the fruit fly diet, which resulted in a shift of the gut microbial composition mainly affecting Lactobacillus, Acetobacter, and Actinobacteria, potentially related to an increased life span and climbing activity, as well as an overexpression of the Spargel gene (PPAR $\gamma$-coactivator $1 \alpha$ (PGC-1 $\alpha)$ homolog) in the male fruit fly [85].

The bacterial diversity and alterations in microbiota dynamics in the fruit fly can be analyzed by using $16 \mathrm{~S}$ rRNA gene sequencing by different methodologies, such as a qPCR approach with species-specific oligonucleotide primer pairs [83], deep gene sequencing approaches using 454 sequencing [38] or whole-genome shotgun sequencing [86], and high-sensitive NanoString nCounter technology for targeted RNA, DNA, or proteins [87]. Initial data also point towards the use of flow cytometric microbiome analysis as an easy-to-use and cost-effective method to unravel effects on the Drosophila microbiota. Although this method does not deliver direct phylogenetic information, it provides information about relative subcommunity abundance and absolute cell numbers at-line through distinct light scatter and fluorescence properties [88]. Staats and colleagues have already used a flow cytometry-based analysis together with the sequencing of the V1-V2 regions of the 16S rRNA to detect changes in the microbiome of Drosophila melanogaster following the intake of the plant bioactive ursolic acid [85].

Drosophila melanogaster has also been demonstrated to be a successful in vivo model system to elucidate the mechanisms of probiotic organisms in the human microbiota (i.e., "beneficial" bacterial species such as Bifidobacterium bifidum) by pathogen inhibition [39, 89]. Recently, the probiotic strain Lactobacillus fermentum NCIMB 5221 and its metabolite ferulic acid have been added to the Drosophila melanogaster larvae medium, targeting the TOR and IIS signaling pathways as well as the larvae's metabolism resulting in an acceleration of its developmental growth [90]. Therefore, elucidating potential molecular pathways of probiotics or its corresponding metabolites by using the fruit fly as a model organism would help to improve therapies for human diseases related to the energy metabolism, such as obesity and diabetes.

\section{Metabolomics}

Metabolomics is referred to a systematic study of detectable small molecules deriving from specific cellular processes in an organism [54]. Metabolomics studies could provide information on the effects of dietary compounds, and their health consequences, on an organism's metabolism. The interpretation of the results is relatively difficult as these metabolites may derive from at least three different sources: (1) from the diet (nutrients/bioactive compounds), (2) from incorporated environmental xenobiotics, and (3) metabolic signals generated by the commensal gut bacteria (microbiota) [54]. The main technologies applied in metabolomics research are mass spectrometry and NMR spectroscopy, both having advantages and disadvantages [59].

Drosophila melanogaster is a well-known model in the context of metabolomics research [91, 92]. However, studies in the context of diet-metabolome interaction are currently very limited. An and Fukusaki [92] provided a list with studies in the fruit fly using metabolomics approaches. Heinrichsen and co-workers [93] analyzed the metabolome of Drosophila melanogaster 
that received a high-fat diet (HFD). In this study, the metabolome of HFD-fed flies showed changes in the metabolism of fatty acids, amino acids, and carbohydrates compared to control diet-fed flies. In another experiment, fruit flies under dietary restriction exhibited different metabolic profiles compared to the corresponding control flies, suggesting a central role of dietary restriction in the prevention of age-associated pathologies [94].

\section{Conclusion and outlook}

Drosophila melanogaster can be established as a well-suited model system in nutrigenomics research due to the fact that it is one of the best-characterized model organisms in genetic research. The fruit fly also offers the possibility to study nutrition-related effects on the genome as the main methods and techniques required are already established. This model organism is also useful for host-microbiota interactions, as Drosophila melanogaster hosts only a small number of bacterial populations in its gut including species also present in the human microbiota. In general, animal welfare ethical review boards do not have to approve experimental settings applying the fruit fly, providing an essential advantage compared to, for example, laboratory rodents [95]. The ideal use of the fruit fly in nutrition and nutrigenomics research would be the establishment of a screening platform delivering essential information on host-genomic interactions. It would assist to discover and validate primary small molecules and narrow it down to the most potential candidates, which may then be tested in rodents and eventually in humans [6]. In this context, Drosophila melanogaster offers additional advantages compared to other research organism models, including a short generation time, a high fecundity, a small genome size, the presence of a high number of genes and conserved metabolic signaling pathways connected with human diseases, a good availability of mutant fly strains, and a relatively cheap maintenance [6]. The fruit fly may also be applied to elucidate the effects of different diets and bioactive compounds, as well as different microbial strains, on the immune system by evaluating the anti-microbial peptide expression in connection with systemic inflammation and gut homeostasis [95, 96]. However, to completely understand the link between genotype, microbiota, and diseases, major obstacles such as the microbial diversity and the genetic complexity of the immune system need to be taken into consideration. The fruit fly gut microbiota can be isolated, cultured, and engineered in a relatively easy way, offering also a convenient model system to analyze microbiota-associated diseases, including metabolic, neurological, and immunological disorders $[16,97]$. The use of Drosophila melanogaster in epigenetics has only been recently introduced and, therefore, needs more research regarding the underlying mechanisms involved in genome stability and regulation $[98,99]$. Additionally, a Drosophila melanogaster model exhibiting specific metabolic deficiencies may be applied to deliver information on dietary and/or pharmaceutic interventions contributing to a personalized nutrition approach prior to a costly testing in a human trial [100]. As recently suggested by Lüersen and colleagues [101], standardized interlaboratory models and protocols for Drosophila melanogaster maintenance are essentially needed which also apply to nutrigenomics research. Finally, this will contribute (a) to the validation of nutrition-based experiments and (b) to a better comparability of nutrition-related studies.

\section{Abbreviations \\ CHD1: Chromatin remodeling factor 1; ChIP: Chromatin immunoprecipitation: CHK: Cell cycle checkpoint kinases; EB: Enteroblast; EC: Enterocyte; \\ EE: Enteroendocrine cell; EGCG: Epigallocatechin gallate; HFD: High-fat diet; IIS: Insulin/insulin-like growth factor signaling; IMD: Immune deficiency; \\ ISC: Intestinal stem cell; JAK-STAT: Janus kinase-signal transducers and \\ activators of transcription; JNK: c-Jun N-terminal kinase; LCA: Lithocholic acid; miRNA: microRNA; NGS: Next-generation sequencing; NMR: Nuclear magnetic resonance; OTU: Operational taxonomic unit; PGC1a: PPARY cO- activator 1a; PM: Peritrophic matrix; QC: Quercetin glycoside; \\ QTL: Quantitative trait loci; rRNA: Ribosomal RNA; TOR: Target of rapamycin; UAS: Upstream activation sequence}

Funding

NB is funded by a postdoctoral grant from the Spanish Foundation "Alfonso Martín Escudero." The funding body had no role in the design and the writing of the manuscript.

Availability of data and materials

Not applicable.

Authors contribution

Both authors drafted the manuscript and read and approved the final manuscript.

Ethics approval and consent to participate

Not applicable.

Consent for publication

Not applicable.

Competing interests

The authors declare that they have no competing interests.

\section{Publisher's Note}

Springer Nature remains neutral with regard to jurisdictional claims in published maps and institutional affiliations.

\section{Author details}

${ }^{1}$ Institute of Nutritional Medicine, University of Lübeck, Ratzeburger Allee 160, 23538 Lübeck, Germany. ${ }^{2}$ Institute of Nutritional Sciences,

Justus-Liebig-University, Wilhelmstrasse 20, 35392 Giessen, Germany.

Received: 5 April 2019 Accepted: 17 April 2019

Published online: 06 May 2019

References

1. Kussmann M, Van Bladeren PJ. The extended nutrigenomics understanding the interplay between the genomes of food, gut microbes, and human host. Front Genet. 2011;21(2). 
2. Ruden DM, De Luca M, Garfinkel MD, Bynum KL, Lu X. Drosophila nutrigenomics can provide clues to human gene-nutrient interactions. Annu Rev Nutr. 2005;25:499-522.

3. Ruden DM, Lu X. Evolutionary conservation of metabolism explains how Drosophila nutrigenomics can help us understand human nutrigenomics. Genes Nutr. 2006;1:75.

4. Reiter LT, Potocki L, Chien S, Gribskov M, Bier E. A systematic analysis of human disease-associated gene sequences in Drosophila melanogaster. Genome Res. 2001;11:1114-25.

5. Rubin GM, Yandell MD, Wortman JR, Gabor Miklos GL, Nelson CR, Hariharan IK, Fortini ME, Li PW, Apweiler R, Fleischmann W, et al. Comparative genomics of the eukaryotes. Science (New York, NY). 2000;287:2204-15.

6. Pandey UB, Nichols CD. Human disease models in Drosophila melanogaster and the role of the fly in therapeutic drug discovery. Pharmacol Rev. 2011; 63:411-36.

7. Ye J, Cui X, Loraine A, Bynum K, Kim NC, White G, De Luca M, Garfinkel MD, Lu X, Ruden DM. Methods for nutrigenomics and longevity studies in Drosophila. In: Tollefsbol TO, Totowa NJ, editors. Biological Aging: Methods and Protocols: Humana Press; 2007. p. 111-41.

8. Parks AL, Cook KR, Belvin M, Dompe NA, Fawcett R, Huppert K, Tan LR, Winter CG, Bogart KP, Deal JE, et al. Systematic generation of highresolution deletion coverage of the Drosophila melanogaster genome. Nat Genet. 2004;36:288-92.

9. Guida MC, Birse RT, Dall'Agnese A, Toto PC, Diop SB, Mai A, Adams PD, Puri $\mathrm{PL}$, Bodmer R. Intergenerational inheritance of high fat diet-induced cardiac lipotoxicity in Drosophila. Nat Commun. 2019;10:193.

10. Hemphill W, Rivera O, Talbert M. RNA-sequencing of Drosophila melanogaster head tissue on high-sugar and high-fat diets. G3 (Bethesda, Md). 2017;8:279-90.

11. Rivera O, McHan L, Konadu B, Patel S, Sint Jago S, Talbert ME. A high-fat diet impacts memory and gene expression of the head in mated female Drosophila melanogaster. J Comp Physiol B. 2019.

12. Oldham S. Obesity and nutrient sensing TOR pathway in flies and vertebrates: functional conservation of genetic mechanisms. Trends Endocrinol Metab. 2011:22:45-52.

13. Smith WW, Thomas J, Liu J, Li T, Moran TH. From fat fruit fly to human obesity. Physiol Behav. 2014:136:15-21.

14. Carreira VP, Mensch J, Hasson E, Fanara JJ. Natural genetic variation and candidate genes for morphological traits in Drosophila melanogaster. PLoS One. 2016;11 e0160069-e69.

15. Chyb S, Gompel N. Atlas of Drosophila morphology: wild-type and classical mutants: Academic Press; 2013.

16. Ugur B, Chen K, Bellen HJ. Drosophila tools and assays for the study of human diseases. Dis Model Mech. 2016:9:235-44.

17. Pool A-H, Scott K. Feeding regulation in Drosophila. Curr Opin Neurobiol. 2014:29:57-63.

18. Lemaitre B, Miguel-Aliaga I. The digestive tract of Drosophila melanogaster. Annu Rev Genet. 2013:47:377-404.

19. Miguel-Aliaga I, Jasper H, Lemaitre B. Anatomy and physiology of the digestive tract of Drosophila melanogaster. Genetics. 2018:210:357-96.

20. Fast D, Duggal A, Foley E. Monoassociation with Lactobacillus plantarum disrupts intestinal homeostasis in adult Drosophila melanogaster. MBio. 2018;9.

21. Li H, Qi Y, Jasper H. Preventing age-related decline of gut compartmentalization limits microbiota dysbiosis and extends lifespan. Cell Host Microbe. 2016;19:240-53.

22. Guo L, Karpac J, Tran SL, Jasper H. PGRP-SC2 promotes gut immune homeostasis to limit commensal dysbiosis and extend lifespan. Cell. 2014; 156:109-22.

23. Clark RI, Salazar A, Yamada R, Fitz-Gibbon S, Morselli M, Alcaraz J, Rana A, Rera M, Pellegrini M, Ja WW, et al. Distinct shifts in microbiota composition during Drosophila aging impair intestinal function and drive mortality. Cell Rep. 2015;12:1656-67.

24. Ren C, Webster P. Finkel SE, Tower J. Increased internal and external bacterial load during Drosophila aging without life-span trade-off. Cell Metab. 2007:6:144-52.

25. Brummel T, Ching A, Seroude L, Simon AF, Benzer S. Drosophila lifespan enhancement by exogenous bacteria. Proc Natl Acad Sci U S A. 2004;101: 12974-9.

26. Douglas AE. Contradictory results in microbiome science exemplified by recent Drosophila research. MBio. 2018;9.

27. Buchon N, Osman D. All for one and one for all: regionalization of the Drosophila intestine. Insect Biochem Mol Biol. 2015;67:2-8.
28. Martin-Duran JM, Hejnol A. The study of Priapulus caudatus reveals conserved molecular patterning underlying different gut morphogenesis in the Ecdysozoa. BMC Biol. 2015;13:29.

29. Kuraishi T, Binggeli O, Opota O, Buchon N, Lemaitre B. Genetic evidence for a protective role of the peritrophic matrix against intestinal bacterial infection in Drosophila melanogaster. Proc Natl Acad Sci U S A. 2011;108: 15966-71.

30. Buchon N, Osman D, David FP, Fang HY, Boquete JP, Deplancke B, Lemaitre B. Morphological and molecular characterization of adult midgut compartmentalization in Drosophila. Cell Rep. 2013;3:1725-38.

31. Peterson CT, Sharma V, Elmen L, Peterson SN. Immune homeostasis, dysbiosis and therapeutic modulation of the gut microbiota. Clin Exp Immunol. 2015:179:363-77.

32. Cuevas-Sierra A, Ramos-Lopez O, Riezu-Boj Jl, Martinez JA, Milagro Fl. Diet, gut microbiota, and obesity: links with host genetics and epigenetics and potential applications. Adv in Nutr. 2019;10:S17-30.

33. Jandhyala SM, Talukdar R, Subramanyam C, Vuyyuru H, Sasikala M, Nageshwar Reddy D. Role of the normal gut microbiota. World J Gastroenterol. 2015:21:8787-803.

34. Clark RI, Walker DW: Role of gut microbiota in aging-related health decline: insights from invertebrate models. Cell Mol Life S. 2018, 75:93-101.

35. Newell PD, Douglas AE. Interspecies interactions determine the impact of the gut microbiota on nutrient allocation in Drosophila melanogaster. Appl Environment Microbiol. 2014;80:788-96.

36. Broderick NA, Lemaitre B. Gut-associated microbes of Drosophila melanogaster. Gut Microbes. 2012;3:307-21.

37. Téfit MA, Leulier F. Lactobacillus plantarum favors the early emergence of fit and fertile adult Drosophila upon chronic undernutrition. J Exp Biol. 2017;220:900-07.

38. Wong CNA, Ng P, Douglas AE. Low-diversity bacterial community in the gut of the fruitfly Drosophila melanogaster. Environ Microbiol. 2011:13:1889-900.

39. Blum JE, Fischer CN, Miles J, Handelsman J. Frequent replenishment sustains the beneficial microbiome of Drosophila melanogaster. MBio. 2013:4:e00860-13.

40. Wong ACN, Chaston JM, Douglas AE. The inconstant gut microbiota of Drosophila species revealed by 16S rRNA gene analysis. SME J. 2013;7:1922.

41. Pais IS, Valente RS, Sporniak M, Teixeira L. Drosophila melanogaster establishes a species-specific mutualistic interaction with stable gutcolonizing bacteria. PLoS Biology. 2018:16 e2005710.

42. Martino ME, Ma D, Leulier F. Microbial influence on Drosophila biology. Curr Opin Microbiol. 2017:38:165-70

43. Heys C, Lize A, Blow F, White L, Darby A, Lewis ZJ. The effect of gut microbiota elimination in Drosophila melanogaster: a how-to guide for hostmicrobiota studies. Ecol Evol. 2018;8:4150-61.

44. Koyle ML, Veloz M, Judd AM, Wong AC, Newell PD, Douglas AE, Chaston $J M$. Rearing the fruit fly Drosophila melanogaster under axenic and gnotobiotic conditions. J Vis Exp. 2016;113: https://doi.org/10.3791/54219.

45. Dobson AJ, Chaston JM, Douglas AE. The Drosophila transcriptional network is structured by microbiota. BMC Genomics. 2016;17:975

46. Keith SA, Eutsey R, Lee H, Solomon B, Oliver S, Kingsford C, Hiller NL, McCartney BM. Identification of microbiota-induced gene expression changes in the Drosophila melanogaster head. bioRxiv. 2019; 561043.

47. Gonzalez MA, Van Booven D, Hulme W, Ulloa RH, Lebrigio RFA, Osterloh J, Logan M, Freeman M, Zuchner S. Whole genome sequencing and a new bioinformatics platform allow for rapid gene identification in $D$. melanogaster EMS screens. Biology. 2012:1:766-77.

48. Everman E, McNeil C, Hackett J, Bain C, Macdonald SJ. Dissection of complex, fitness-related traits in multiple Drosophila mapping populations offers insight into the genetic control of stress resistance. bioRxiv. 2019; 383802.

49. Dietzl G, Chen D, Schnorrer F, Su KC, Barinova Y, Fellner M, Gasser B, Kinsey K, Oppel S, Scheiblauer S, et al. A genome-wide transgenic RNAi library for conditional gene inactivation in Drosophila. Nature. 2007;448:151-6.

50. Cho KS, Bang SM, Toh A. Chapter 26 - Lipids and lipid signaling in drosophila models of neurodegenerative diseases. In: Watson RR, De Meester F, editors. Omega-3 fatty acids in brain and neurological health: Boston: Academic Press; 2014. p. 327-36.

51. Brosius J. The fragmented gene. Ann N Y Acad Sci. 2009:1178:186-93.

52. Schwartz YB, Cavalli G. Three-dimensional genome organization and function in Drosophila. Genetics. 2017:205:5-24.

53. Duffus $\mathrm{H}$, Nordberg $M$, Templeton DM. Glossary of terms used in toxicology, 2nd edition - (IUPAC recommendations 2007). Pure Appl Chem. 2007;79:1153-344 
54. Ferguson LR, Barnett MP. Why are omics technologies important to understanding the role of nutrition in inflammatory bowel diseases? Int J Mol Sci. 2016;17.

55. Azuma M, Dat Le T, Yoshimoto $Y$, Hiraki N, Yamanaka M, Omura F, Inoue $\mathrm{YH}$. RNA-seq analysis of diet-driven obesity and anti-obesity effects of quercetin glucoside or epigallocatechin gallate in Drosophila adults. Eur Rev Med Pharmacol Sci. 2019;23:857-76.

56. Staats S, Rimbach G, Kuenstner A, Graspeuntner S, Rupp J, Busch H, Sina C Ipharraguerre IR, Wagner AE. Lithocholic acid improves the survival of Drosophila melanogaster. Mol Nutr Food Res. 2018;62:e1800424.

57. Li L, Edgar BA, Grewal SS. Nutritional control of gene expression in Drosophila larvae via TOR, Myc and a novel cis-regulatory element. BMC Cell Biol. 2010;11:7.

58. Wagner AE, Terschluesen AM, Rimbach G. Health promoting effects of brassica-derived phytochemicals: from chemopreventive and antiinflammatory activities to epigenetic regulation. Oxid Med Cell Longev. 2013:2013:964539.

59. Verma M, Khoury MJ, loannidis JP. Opportunities and challenges for selected emerging technologies in cancer epidemiology: mitochondrial, epigenomic, metabolomic, and telomerase profiling. Cancer Epidemiol Biomarkers Prev. 2013;22:189-200.

60. Lumey LH, Stein AD, Kahn HS, van der Pal-de Bruin KM, Blauw GJ, Zybert PA, Susser ES. Cohort profile: the Dutch Hunger Winter families study. Int J Epidemiol. 2007;36:1196-204.

61. Ravelli AC, van der Meulen JH, Michels RP, Osmond C, Barker DJ, Hales CN, Bleker OP. Glucose tolerance in adults after prenatal exposure to famine. Lancet. 1998;351:173-7.

62. Heijmans BT, Tobi EW, Stein AD, Putter H, Blauw GJ, Susser ES, Slagboom PE, Lumey LH. Persistent epigenetic differences associated with prenatal exposure to famine in humans. Proc Natl Acad Sci U S A. 2008;105:17046-9.

63. Tobi EW, Goeman JJ, Monajemi R, Gu H, Putter H, Zhang Y, Slieker RC, Stok AP, Thijssen PE, Muller F, et al. DNA methylation signatures link prenatal famine exposure to growth and metabolism. Nat Commun. 2014;5:5592.

64. Osborne AJ, Dearden PK. A 'phenotypic hangover': the predictive adaptive response and multigenerational effects of altered nutrition on the transcriptome of Drosophila melanogaster. Environ Epigenet. 2017;3:dvx019.

65. Lian T, Gaur U, Wu QI, Tu J, Sun B, Yang D, Fan X, Mao X, Yang M. DNA methylation is not involved in dietary restriction induced lifespan extension in adult Drosophila. Genet Res (Camb). 2018;100:e1.

66. Akagi K, Wilson KA, Katewa SD, Ortega M, Simons J, Hilsabeck TA, Kapuria S, Sharma A, Jasper H, Kapahi P. Dietary restriction improves intestinal cellular fitness to enhance gut barrier function and lifespan in D. melanogaster. PLoS Genet. 2018;14:e1007777.

67. Sebald J, Willi M, Schoberleitner I, Krogsdam A, Orth-Holler D, Trajanoski Z, Lusser A. Impact of the chromatin remodeling factor chd1 on gut microbiome composition of Drosophila melanogaster. PLoS One. 2016;11:e0153476.

68. Bibbo S, laniro G, Giorgio V, Scaldaferri F, Masucci L, Gasbarrini A, Cammarota G. The role of diet on gut microbiota composition. Eur Rev Med Pharmacol Sci. 2016;20:4742-9.

69. Brown K, DeCoffe D, Molcan E, Gibson DL. Diet-induced dysbiosis of the intestinal microbiota and the effects on immunity and disease. Nutrients. 2012:4:1095-119.

70. Sturm C, Wagner AE. Brassica-derived plant bioactives as modulators of chemopreventive and inflammatory signaling pathways. Int J Mol Sci. 2017;18.

71. Pal AS, Kasinski AL. Animal Models to Study MicroRNA Function. Adv Cancer Res. 2017;135:53-118.

72. Boesch-Saadatmandi C, Loboda A, Wagner AE, Stachurska A, Jozkowicz A, Dulak J, Doring F, Wolffram S, Rimbach G. Effect of quercetin and its metabolites isorhamnetin and quercetin-3-glucuronide on inflammatory gene expression: role of miR-155. J Nutr Biochem. 2011;22:293-9.

73. Boesch-Saadatmandi C, Wagner AE, Wolffram S, Rimbach G. Effect of quercetin on inflammatory gene expression in mice liver in vivo - role of redox factor 1, miRNA-122 and miRNA-125b. Pharmacol Res. 2012;65: 523-30.

74. Wagner AE, Boesch-Saadatmandi C, Dose J, Schultheiss G, Rimbach G. Antiinflammatory potential of allyl-isothiocyanate--role of Nrf2, NF-(kappa) B and microRNA-155. J Cell Mol Med. 2012;16:836-43.

75. Lee RC, Feinbaum RL, Ambros V. The C. elegans heterochronic gene lin-4 encodes small RNAs with antisense complementarity to lin-14. Cell. 1993;75: 843-54.
76. Kondo S, Ueda R. Highly improved gene targeting by germline-specific Cas 9 expression in Drosophila. Genetics. 2013;195:715-21.

77. Baynam G, Walters M, Claes P, Kung S, LeSouef P, Dawkins H, Bellgard M, Girdea M, Brudno M, Robinson P, et al. Phenotyping: targeting genotype's rich cousin for diagnosis. J Paediatr Child Health. 2015;51:381-6.

78. Li HM, Margam V, Muir WM, Murdock LL, Pittendrigh BR. Changes in Drosophila melanogaster midgut proteins in response to dietary BowmanBirk inhibitor. Insect Mol Biol. 2007;16:539-49.

79. Culwell TF, Thulin CD, Merrell KJ, Graves SW. Influence of diet on the proteome of Drosophila melanogaster as assessed by two-dimensional gel electrophoresis and capillary liquid chromatography-mass spectrometry: the hamburger effect revisited. J Biomol Tech. 2008;19:244-50.

80. Garber K. Debate rages over proteomic patterns. J Natl Cancer Inst. 2004;96: $816-8$.

81. Loch G, Zinke I, Mori T, Carrera P, Schroer J, Takeyama H, Hoch M Antimicrobial peptides extend lifespan in Drosophila. PloS One. 2017;12: e0176689-e89.

82. Hanson MA, Dostalova A, Ceroni C, Poidevin M, Kondo S, Lemaitre B. Synergy and remarkable specificity of antimicrobial peptides in vivo using a systematic knockout approach. Elife. 2019;8.

83. Fink C, Staubach F, Kuenzel S, Baines JF, Roeder T. Noninvasive analysis of microbiome dynamics in the fruit fly Drosophila melanogaster. Appl Environ Microbiol. 2013;79:6984-8.

84. Erkosar B, Yashiro E, Zajitschek F, Friberg U, Maklakov AA, van der Meer JR, Kawecki TJ. Host diet mediates a negative relationship between abundance and diversity of Drosophila gut microbiota. Ecol Evol. 2018;8:9491-502.

85. Staats S, Wagner AE, Luersen K, Kunstner A, Meyer T, Kahns AK, Derer S, Graspeuntner S, Rupp J, Busch H, et al. Dietary ursolic acid improves health span and life span in male Drosophila melanogaster. Biofactors. 2019:45:169-86

86. Petkau K, Fast D, Duggal A, Foley E. Comparative evaluation of the genomes of three common Drosophila-associated bacteria. Biol Open. 2016; 5:1305-16.

87. Fulga TA, McNeill EM, Binari R, Yelick J, Blanche A, Booker M, Steinkraus BR, Schnall-Levin M, Zhao Y, DeLuca T, et al. A transgenic resource for conditional competitive inhibition of conserved Drosophila microRNAs. Nat Commun. 2015:6:7279.

88. Lambrecht J, Schattenberg F, Harms H, Mueller S. Characterizing microbiome dynamics - flow cytometry based workflows from pure cultures to natural communities. J Vis Exp. 2018;137. https://doi.org/10.3791/5803.

89. Neyen C, Bretscher AJ, Binggeli O, Lemaitre B. Methods to study Drosophila immunity. Methods. 2014;68:116-28.

90. Westfall S, Lomis N, Prakash S. Ferulic Acid Produced by Lactobacillus fermentum Influences Developmental Growth Through a dTOR-Mediated Mechanism. Molecular Biotechnology. 2019;61:1-11.

91. Cox JE, Thummel CS, Tennessen JM. Metabolomic Studies in Drosophila. Genetics. 2017;206:1169-85.

92. An PNT, Fukusaki E. Metabolomics: State-of-the-Art Technologies and Applications on Drosophila melanogaster. Drosophila Models for Human Diseases. 2018:1076:257-76.

93. Heinrichsen ET, Zhang H, Robinson JE, Ngo J, Diop S, Bodmer R, Joiner WJ, Metallo CM, Haddad GG. Metabolic and transcriptional response to a highfat diet in Drosophila melanogaster. Mol Metab. 2014;3:42-54.

94. Laye MJ, Tran V, Jones DP, Kapahi P, Promislow DE. The effects of age and dietary restriction on the tissue-specific metabolome of Drosophila. Aging Cell. 2015:14:797-808.

95. Trinder M, Daisley BA, Dube JS, Reid G: Drosophila melanogaster as a highthroughput model for host-microbiota interactions. Front Microbiol. 2017;8: 751-51.

96. Staats S, Luersen K, Wagner AE, Rimbach G. Drosophila melanogaster as a versatile model organism in food and nutrition research. J Agric Food Chem. 2018:66:3737-53.

97. Apidianakis Y, Rahme LG. Drosophila melanogaster as a model for human intestinal infection and pathology. Dis Model Mech. 2011;4:21-30.

98. Solovev I, Shaposhnikov M, Kudryavtseva A, Moskalev A. Chapter 14 Drosophila melanogaster as a model for studying the epigenetic basis of aging. In: Moskalev A, Vaiserman AM, editors. Epigenetics of aging and longevity. Boston: Academic Press; 2018. p. 293-307.

99. Wood JG, Jones BC, Jiang N, Chang C, Hosier S, Wickremesinghe P, Garcia M, Hartnett DA, Burhenn L, Neretti N, et al. Chromatin-modifying genetic interventions suppress age-associated transposable element activation and extend life span in Drosophila. Proc Natl Acad Sci U S A. 2016;113:11277-82. 
100. Ruden DM, Rasouli P, Wang L, Lu X. Nutrient-by-genotype interactions and personalized diet: what can we learn from Drosophila and evolutionary biology? Curr Pharmacogenomics Personal Med. 2009;7:189-204.

101. Lüersen K, Röder T, Rimbach G. Drosophila melanogaster in nutrition research - the importance of standardizing experimental diets. Genes Nutr. 2019;14:3

Ready to submit your research? Choose BMC and benefit from:

- fast, convenient online submission

- thorough peer review by experienced researchers in your field

- rapid publication on acceptance

- support for research data, including large and complex data types

- gold Open Access which fosters wider collaboration and increased citations

- maximum visibility for your research: over $100 \mathrm{M}$ website views per year

At $B M C$, research is always in progress.

Learn more biomedcentral.com/submissions 